\title{
Bacterial nanotubes for intimate sharing
}

\author{
Mitesh Dongre ${ }^{1,2}$, Bernt Eric Uhlin ${ }^{1,2,3}$ and Sun Nyunt Wai, ${ }^{1 *}$ \\ 1 Department of Molecular Biology, Umeå University, Umeå, Sweden \\ 2 Umeå Centre for Microbial Research, Umeå University, Umeå, Sweden \\ ${ }^{3}$ The Laboratory for Molecular Infection Medicine Sweden, Umeå University, Umeå, Sweden \\ *Correspondence: sun.nyunt.wai@molbiol.umu.se
}

\section{A commentary on}

Intercellular nanotubes mediate bacterial communication

by Dubey, G. P., and Ben-Yehuda, S. (2011). Cell 144, 590-600.

Classical genetics and biochemical studies on bacterial communication have established that bacteria can exchange small circular plasmids and DNA fragments via conjugative pili or through the agency of a transducing phage. Additionally, homogenous and heterogeneous bacterial population can share chemical information by secreting pheromone-like molecules called as QS auto-inducers or by discharging cytoplasmic and periplasmic proteins, antimicrobial factors, and even DNA in packaged outer membrane vesicles (MVs), which can deliver the cargo to distant cells. Delivery of cellular proteins via a direct cellular contact is also not uncommon in bacteria. Many pathogenic Gram negative bacteria can deliver cytoplasmic effector proteins directly into the host cytoplasm using specialized secretory systems. These secretion systems can employ highly orchestrated needle complexes as in the type III secretion system (T3SS) and the type VI secretion system (T6SS) or pilin channels as in the type IV secretion system, for the delivery of toxins and effector molecules directly into host (Tseng et al., 2009). However, these secretory systems are principally unidirectional delivery apparatus ensembling unique constituent proteins in a highly energy expensive process. Nonetheless, there has been no evidence of bacteria sharing large proteins and DNA fragments through direct cytoplasmic contact.

The recent report by Dubey and BenYehuda (2011) in Cell incepted and evidenced the presence of intercellular membranous bridges or nanotubes between proximal bacteria of same as well as different species taking Bacillus subtilis,
Streptococcus pyogenes, and Escherichia coli as model Gram positive and Gram negative organisms. This discovery has opened an interesting new arena in the field of bacterial communication.

In their study the team characterized hereditary transfer of non-conjugative plasmids and non-hereditary transfer of cytoplasmic traits such as green fluorescent protein (GFP) and antimicrobial resistance protein between neighboring bacteria which was dependent on relative distance between the cells and time. Through high resolution scanning electron microscopy they observed the intercellular membranous conduits between the bacterial cells, facilitating direct cytoplasmic sharing between adjacent bacterial cells.

Further, by analyzing thin sections EM of the nanotubes and their disruption by SDS, authors enunciated that these conduits may involve direct cytoplasmic fusions of the adjacent bacteria secured by a multilayer structure comprising cell wall and plasma membrane. This contingency could make these nanotubes as unique rendezvous points between two bacterial cells distinct from other secretory mechanisms in that they may not involve any dedicated cellular machinery for the transfer of cytoplasmic material. Moreover, the diameter of these nanotubes was observed at more than $100 \mathrm{~nm}$ which is larger than most pili and fimbriae and is sufficiently large to allow transfer of large protein complexes and DNA fragments.

It is interesting to note that most of these features of nanotubes are pertinent to MVs which frequently emerge from the cell wall of Gram negative and Gram positive bacteria. For instance, MVs are membranous structure measuring $20-300 \mathrm{~nm}$ in diameter, emerging from the cell wall of Gram negative as well as Gram positive bacteria and carry periplasmic and cytoplasmic proteins, RNA, and DNA fragments along with intrinsic membrane proteins (Wai et al.,
2003; Mashburn-Warren and Whiteley, 2006; Lee et al., 2009). Biogenesis of MVs in Gram negative bacteria is not random blebbing of the outer membrane but in fact occur at specific cell surface points excluding the abundant Braun lipoprotein which traverse through outer membrane and peptidoglycan layer (Hoekstra et al., 1976). Moreover, a recent study revealed that in Pseudomonas aeruginosa MVs primarily contain highly charged B-band LPS which is relatively rare in comparison with the abundant A-band LPS in OM (Li et al., 1996). It is proposed that localization of these charged moieties at unlinked areas coupled with the accumulation of cargo proteins and some unidentified curvatureinducing molecules in the periplasm build a substantial inducing force beneath the outer membrane causing an outward curvature which culminates in release of MVs (Kulp and Kuehn, 2010). Further, due to the intrinsic bilayered exterior, these MVs can readily adhere to and fuse with cell wall of other bacteria.

Incidentally, Dubey and Ben-Yehuda (2011) also observed similar membrane bulging during initiation of the nanotubes formation. Given the degree of similarity between MVs and nanotubes it is plausible that initiation of these conduits might require similar framework of preferential biogenesis sites on the bacterial cell wall excluding specific membrane components or including preferential cargo which would act as inducing force. Moreover, since nanotube formation is governed by relative distance between two adjacent bacteria, it is possible that these conduits are formed by fusion of two budding MVs from respective bacteria which otherwise would shed these vesicles if not in close proximity of each other. Another fact which stipulate resemblance between nanotubes and MVs is that both allows concurrent delivery of multiple secreted molecules to the host at higher concentration which could be a prerequisite for certain bioprocesses. 
A major relevance of the discovery of nanotubes is that it can help in improving our understanding about the communication between the inhabitants and accretion of hereditary and non-hereditary traits by constituent bacteria in single and multispecies biofilms. Moreover, since MVs are shown to be a common particulate constituent of the matrix of many Gram negative and multispecies biofilms (Schooling and Beveridge, 2006), it is of further interest to study the correlation between these two coherently analogous modes of bacterial secretion.

\section{REFERENCES}

Dubey, G. P., and Ben-Yehuda, S. (2011). Intercellular nanotubes mediate bacterial communication. Cell 144, 590-600.

Hoekstra, D., Van Der Laan, J. W., De Leij, L., and Witholt, B. (1976). Release of outer membrane fragments from normally growing Escherichia coli. Biochim. Biophys. Acta 455, 889-899.

Kulp, A., and Kuehn, M. J. (2010). Biological functions and biogenesis of secreted bacterial outer membrane vesicles. Annu. Rev. Microbiol. 64, 163-184.

Lee, E. Y., Choi, D. Y., Kim, D. K., Kim, J. W., Park, J. O., Kim, S., Kim, S. H., Desiderio, D. M., Kim, Y. K., Kim, K. P., and Gho, Y. S. (2009). Gram-positive bacteria produce membrane vesicles: proteomics-based characterization of Staphylococcus aureus-derived membrane vesicles. Proteomics 9, 5425-5436.

Li, Z., Clarke, A. J., and Beveridge, T. J. (1996). A major autolysin of Pseudomonas aeruginosa: subcellular distribution, potential role in cell growth and division and secretion in surface membrane vesicles. J. Bacteriol. 178, 2479-2488.

Mashburn-Warren, L. M., and Whiteley, M. (2006). Special delivery: vesicle trafficking in prokaryotes. Mol. Microbiol. 61, 839-846.

Schooling, S. R., and Beveridge, T. J. (2006). Membrane vesicles: an overlooked component of the matrices of biofilms. J. Bacteriol. 188, 5945-5957.

Tseng, T. T., Tyler, B. M., and Setubal, J. C. (2009). Protein secretion systems in bacterial-host asso- ciations, and their description in the Gene Ontology. BMC Microbiol. 9(Suppl. 1), S2. doi: 10.1186/1471-2180-9-S1-S2

Wai, S. N., Lindmark, B., Soderblom, T., Takade, A., Westermark, M., Oscarsson, J., Jass, J., RichterDahlfors, A., Mizunoe, Y., and Uhlin, B. E. (2003). Vesicle-mediated export and assembly of poreforming oligomers of the enterobacterial ClyA cytotoxin. Cell 115, 25-35.

Received: 01 May 2011; accepted: 02 May 2011; published online: 11 May 2011.

Citation: Dongre M, Uhlin BE and Wai SN (2011) Bacterial nanotubes for intimate sharing. Front. Microbio. 2:108. doi: 10.3389/fmicb.2011.00108

This article was submitted to Frontiers in Cellular and Infection Microbiology, a specialty of Frontiers in Microbiology.

Copyright (C) 2011 Dongre, Uhlin and Wai. This is an openaccess article subject to a non-exclusive license between the authors and Frontiers Media SA, which permits use, distribution and reproduction in other forums, provided the original authors and source are credited and other Frontiers conditions are complied with. 